\title{
BMJ Open Negative recollections regarding doctor- patient interactions among men receiving a prostate cancer diagnosis: a qualitative study of patient experiences in Japan
}

Masako Torishima (D) , ${ }^{1,2}$ Michiko Urao, ${ }^{2}$ Takeo Nakayama, ${ }^{3}$ Shinji Kosugi ${ }^{1,2}$

To cite: Torishima M, Urao M, Nakayama T, et al. Negative recollections regarding doctorpatient interactions among men receiving a prostate cancer diagnosis: a qualitative study of patient experiences in Japan. BMJ Open 2020;10:e032251. doi:10.1136/ bmjopen-2019-032251

- Prepublication history and additional material for this paper are available online. To view these files, please visit the journal online (http://dx.doi. org/10.1136/bmjopen-2019032251).

Received 10 June 2019 Revised 23 October 2019 Accepted 11 November 2019

D) Check for updates

(c) Author(s) (or their employer(s)) 2020. Re-use permitted under CC BY-NC. No commercial re-use. See rights and permissions. Published by BMJ.

${ }^{1}$ Clinical Genetics Unit, Kyoto University Hospital, Kyoto, Sakyo-ku, Japan

${ }^{2}$ Department of Medical Ethics and Medical Genetics, Kyoto University School of Public Health, Kyoto, Sakyo-ku, Japan ${ }^{3}$ Department of Health Informatics, Kyoto University School of Public Health, Kyoto, Sakyo-ku, Japan

Correspondence to Mrs Masako Torishima; mtori@kuhp.kyoto-u.ac.jp

\section{ABSTRACT}

Objectives To explore the negative recollections of prostate cancer patients regarding the attitudes and language used by the doctors in delivering their diagnoses in Japan, in order to improve patient-centred communication.

Design and setting This is a qualitative secondary analysis of the prostate cancer narrative data from the Database of Individual Patients' Experiences-Japan archives. A thematic analysis was conducted regarding negative recollections of doctors' words/attitudes when delivering a cancer diagnosis. Recruitment was based on maximum variation sampling. Participants were recruited from medical institutions, patient associations and through media advertisements.

Participants Men with a diagnosis of prostate cancer $(n=51)$.

Findings 0 the 51 participants, 17 had negative recollections of the doctors' words/attitudes during the delivery of the cancer diagnosis. After thematic analysis, 11 categories emerged: 'Surprised by the abrupt disclosure of the diagnosis', 'Displeased by the direct disclosure of the diagnosis to the patient in the absence of family members', 'Unable to accept the doctor's negative words in the explanations', 'Unable to understand the doctor's technical jargon', 'Distrust due to failure in diagnosis based on previous examinations', 'Aggrieved at the doctor's unwillingness to allow questions', 'Dissatisfied with explanations involving facts only', 'Indignant over the unexpected disclosure of life expectancy', 'Unable to accept the doctor's blame for the delay in the initial hospital visit', 'Uncomfortable with the usage of inappropriate metaphors' and 'Pessimistic thoughts despite optimistic explanations'. Conclusions It is clear that patients have recollections of a variety of negative experiences regarding the words/ attitudes of their doctors at the time they received their prostate cancer diagnosis. Thus, the use of narrative data would facilitate the appropriate application of commonly used guidelines for the delivery of cancer diagnoses tailored to individual patients in clinical practice.

\section{INTRODUCTION}

Prostate cancer is the second most common cancer among men in the world. ${ }^{2}$ The
Strengths and limitations of this study

- Data from the Database of Individual Patients Experiences (DIPEx)-Japan data archives were compiled using the rigorous methodology developed by the Health Experience Research Group of the University of Oxford, UK.

- A maximum variation sampling method was applied to the DIPEx data archives.

- The patients' negative recollections regarding the delivery of their cancer diagnosis need to be remedied, given their clinical importance.

- Since patient sampling was not specific to the current research theme, it is possible that variations in the patients' negative recollections did not reach theoretical saturation.

- All participants in this study were Japanese; thus, generalisability to other cultures/countries remains unclear.

incident rate is increasing in Asian countries such as Japan, Singapore and Korea. ${ }^{3-6}$ The global age-standardised incidence and mortality rates were 29.3 and 7.6 in $2018 .^{2}$ On the other hand, in Japan, in 2018, agestandardised incidence and mortality rates were 35.4 and $4.4 .^{7}$ Another survey reported the 5 -year survival rate at almost $100 \% .{ }^{8}$ Prostate cancer has a relatively favourable prognosis; thus, the quality of life after diagnosis is an important consideration.

While there has been an increasing body of qualitative research based on narratives provided by prostate cancer patients mainly in the USA and Western countries, ${ }^{9-13}$ reports from Asia have been scarce. ${ }^{1415}$ Some reports suggest that being informed of a cancer diagnosis can have an effect on patients' mental health, which may vary depending on how the diagnosis was delivered. ${ }^{16}{ }^{17}$ Therefore, the patients' understanding and satisfaction 
with the doctor-patient dialogue and their general trust in the doctor may have an effect on health. ${ }^{18}$ As such, the words and attitude of the doctor when breaking the bad news of a cancer diagnosis to a patient are critical to the patient's life.

The SPIKES guidelines (online supplementary figure 1 ), as recommended by the American Society of Clinical Oncology, ${ }^{19}$ are widely used in many countries for the delivery of cancer diagnoses. However, the SPIKES protocol was developed based mainly on the opinions of oncologists; there is insufficient investigation into the patient's point of view. However, patient-doctor communication is affected by cultural factors. ${ }^{20-22}$ In Japan, the SHARE protocol (online supplementary figure 2) was developed in 2005 by surveying cancer patients about their notions of an ideal conversation when receiving news of cancer diagnosis. ${ }^{23-26}$ SHARE includes the important item, 'the doctor should encourage the patient to ask questions', ${ }^{27}$ which is absent in SPIKES. SHARE has been promoted by the Japan Psycho-Oncology Society and is widely used in Japan. These communication protocols have important factors in common regarding the delivery of cancer diagnoses and are not limited by the type of cancer. However, according to patients, guidelines suited to the specific type of cancer are needed in order to deliver cancer diagnoses in a more ideal way. It is important to elucidate the actual experiences-especially the negative experiences-of patients receiving their cancer diagnosis in order to create guidelines suited to each cancer type.

Thus, we aimed to explore the negative recollections of cancer patients about doctors' words/attitudes during the delivery of a cancer diagnosis. This study focused on prostate cancer because there are insufficient numbers of practical studies conducted in Japan, despite the high burden of patients with prostate cancer.

\section{METHODS}

\section{Study design}

This study was a qualitative secondary analysis ${ }^{28} 29$ conducted to evaluate prostate cancer patients' narratives drawn from an existing database. The interview text was read several times, and a thematic analysis was conducted of the negative recollections of patients about doctors' words/attitudes during the delivery of cancer diagnoses.

\section{Data collection}

DIPEx International (http://www.dipexinternational. $\mathrm{org} /$ ) promotes the acquisition/utilisation of narrative data based on the concept of 'patient-centred medical care implementation', with a focus on the importance of 'talking about health and illness' as a social resource in accordance with the qualitative research methods developed at Oxford University in the UK. ${ }^{30}{ }^{31}$ DIPEx-Japan (https://www.dipex-j.org/) is a member organisation of DIPEx International; it classifies the collected stories by theme using the strictly formulated methods of the organisation and publishes a sampling of videos, voice files and text files online for free. The content of the recorded interviews in the DIPEx-Japan narrative data published online comprises a small portion $(10 \%-20 \%)$ of the total information. ${ }^{15}$ The original interview data, including the portions not published online, are compiled into a narrative data archive that can be used for secondary research. The DIPEx-Japan interviewers take courses at Oxford University in the UK on data collection/analysis and web design.

The DIPEx-Japan prostate cancer narrative data contains interviews with 51 Japanese prostate cancer patients. The selection criteria for participants were as follows: (i) diagnosed more than 6 months ago, (ii) not currently hospitalised and (iii) of over 20 years of age. Recruitment took place through calls to designated cancer hospitals and prostate cancer patient associations across Japan, and maximum variation sampling method was used to achieve the diversity of experiences based on age, structure of the patient's family and place of residence, symptoms and the type of treatment or care. The data collection period was from 30 December 2007 to 30 January 2015.

The interview format was a combination of unstructured and semi-structured interview methods. At the start of the interview, an open-ended question was asked to begin the unstructured section: 'Can you tell me about your experience with cancer from the time you first noticed something unusual?'. In a slightly different manner than usual for the typical qualitative research, with consideration for ensuring that there was a website that could be easy to read and listen to, the interviewer used a variety of nonverbal prompts such as nodding, expressions and eye contact to avoid voices overlapping. Near the end of the interviewee's illness story, the interviewer would start asking questions to supplement his story. The interview guide contained a wide range of topics such as: the sentiment they experienced on disclosure of their disease; details of selecting a hospital or treatment; the treatment selection process; and the physical/mental/economic impact of the diagnosis and treatments. However, not all of the earlier questions were necessarily asked in each individual interview.

In this study, we used verbatim records from the data archives created for the paid data sharing system provided by DIPEx-Japan, which includes the portion that is not published online. We focused on the 'scene when first receiving the cancer diagnosis', that is, the scene when a doctor first revealed a cancer diagnosis to the patient, but excluded the scene in which the doctor explained the treatments, etc., even if it was done on the same day.

This study was a secondary analysis using DIPEx-Japan data archives. Both the researchers for this study and DIPEx-Japan researchers obtained ethical approval. DIPEx-Japan obtained approval from the Osaka Prefecture University School of Nursing Research Ethics Committee (21 55). 
Consent for use of the narrative data (to compile narrative archives to be put to secondary use) was obtained at the time of the interview. All words that could identify individuals or which the interviewees requested not to be recorded were deleted.

\section{Patient and public involvement}

No patients were involved, since this study was a secondary analysis using the narrative data archives of DIPEx-Japan.

\section{Data analysis}

The data were analysed using inductive thematic analysis. ${ }^{32} 33$ The procedure of the main analysis was as follows:

1. The researcher (MT) read the data from all 51 patients from the data archives several times.

2. MT extracted the narrative data referring to the patients' recollections of the words/attitude of the doctor during delivery of the cancer diagnosis.

3. The extracted data were coded and labelled by MT, sentence by sentence.

4. MT grouped the labels by similarity and assigned the groups subcategory names.

5. MT extracted the subcategories referring to negative recollections from all subcategories.

6. MT grouped the subcategories by similarity and gave the groups category names.

7. For steps 2 and 5, MT and a co-author (MU) then investigated whether the scope of the extraction was valid. From steps 2 to 6, the two authors (MT and MU) discussed the validity of each name (label, subcategory, and category) until a consensus was reached. Finally, the analytical results from the discussions of MT and MU from steps 3 to 6 were re-examined by MT, MU and TN.

MT (BSN, MPH) has a 6-year experience as a certified genetic counsellor and conducted qualitative research during her master's degree programme.

MU (BA, MPH) is a counsellor/certified psychologist with over 30 years' experience and has been involved in qualitative research for 17 years, both as a researcher and as a lecturer.

$\mathrm{TN}(\mathrm{MD}, \mathrm{PhD})$ is a physician and an epidemiologist of over 30 years, and he has multiple supervisory experiences with qualitative research theses. He established DIPEx-Japan.

\section{FINDINGS}

\section{Data description}

The narrative data for 51 prostate cancer patients were studied. Their age ranged from 52 to 85 years. The period between diagnosis and interview ranged from 6 months to 16 years. The patients were diagnosed between 1992 and 2014, with most cases occurring between 2005 and 2008. The interview dates ranged from 2007 to 2015, with most of them conducted in 2008. The interview location was at home for 36 of the 51 patients. All of the interviews were audio-recorded, and 46 participants who agreed to appear on the web were video-recorded as well. The word count for all 51 prostate cancer patients' narrative data was 1946083 words. The code count of 'scene when first receiving the cancer diagnoses' was 167 codes. The number of codes of negative recollections regarding prostate cancer diagnosis was 42 of $167(25.1 \%)$ codes; 11 categories were extracted from 42 codes. The characteristics of the 17 men with negative recollections regarding cancer diagnosis and those for all 51 men are shown in table 1 . Seventeen patients with negative recollections were diagnosed between 2001 and 2007; 5 of the $17(29.4 \%)$ patients had advanced cancer compared with the 6 of $51(11.8 \%)$ patients overall.

\section{Patients' negative recollections of their doctor's words/ attitude while receiving the diagnosis}

Of the 51 patients in this study, 17 spoke of negative recollections of the doctor's words or attitudes. The categories were brought to the conceptual level, yielding a total of 11 categories (box 1). In the narrative data (made up of interviews that were not focused on negative experiences regarding their cancer diagnosis), if the research had been focused specifically on negative experiences initially, patients would have likely spoken even more about a diverse range of negative experiences. The negative words/attitudes of doctors that remained in the patients' memories were not necessarily obviously problematic words or attitudes. The coding tree will be available as online supplementary figure 3 .

Surprised at the abrupt disclosure of the diagnosis

Patients believed that the disclosure of cancer diagnosis was a highly sensitive issue, and they expected that the doctor might say, 'This may be cancer' as a preliminary step in the delivery of the cancer diagnosis. However, contrary to their expectation, doctor's way of disclosure caused the patient felt that the cancer diagnosis information came too suddenly.

Displeased by direct disclosure of the diagnosis to the patient in the absence of family members

One patient believed that doctors should give cancer notifications when the patient has his spouse or other family members with him, not when he is alone. He was displeased that the diagnosis was delivered to him directly and casually, without the presence of family members (diagnosed in 2001). When a patient feels that 'cancer is scary', such casual notification leaves a negative impression in the memory.

Unable to accept the doctor's negative words in the explanations

Patients had negative recollections of the doctor's words used in the explanation of the malignant nature of their cancer such as 'bad-looking', 'the worst kind', and 'a very serious figure'.

Unable to understand the doctor's technical jargon

When the doctor used medical terminology, for example, invasive cancer or well-differentiated cancer, etc., patients were left with the sense that they had not understood anything at all. 
Table 1 Characteristics of the data sample

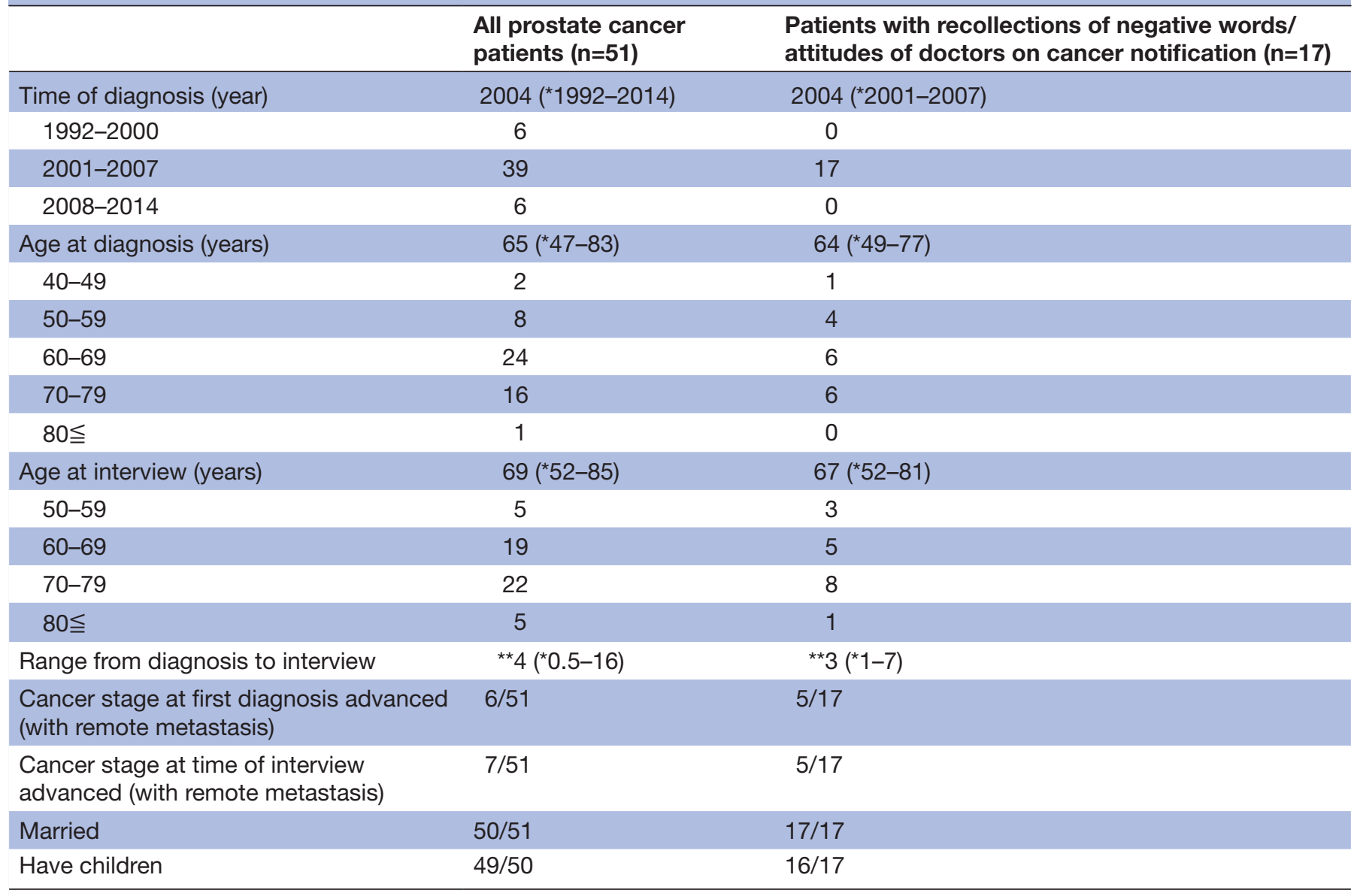

*range (minimum-maximum); **median.

Distrust due to failure in the diagnosis based on previous examinations

The doctor said to the patient, 'The biopsy revealed cancer cells on the opposite side of the previous biopsy'. The patient distrusted the doctor because the cancer should have been identified on CT but was not.

Aggrieved at doctor's unwillingness to allow questions

Even though the patient tried to ask questions after hearing the diagnosis, the doctor urged him to leave without giving him any time, such as 'Oh, um, take care, take care, take care', which was more like get out of here, get out of here, get out of here. The patient was left with a sense of dissatisfaction.

Dissatisfied with explanations involving facts only

When doctors dispassionately listed off medical facts alone and do not take into consideration the patient's feelings. When a doctor told a patient, 'You only have a few months left to live', the patient asked, 'Huh? What do you mean?' in fright, and the doctor proceeded to explain that, "the cancer has metastasised throughout your entire body'. Clearly, this encounter left the patient with a negative memory. He remarked 'When I received the cancer diagnosis, I panicked and said "What?". I was disappointed when the doctor responded with only factual medical explanations'.
Indignant over the unexpected disclosure of life expectancy

Patients who were unexpectedly informed of their limited life expectancy while they were still in the shock of having heard a cancer diagnosis were left with a particularly bitter memory. Unexpected disclosure of life expectancy is like that. A patient asked, 'About your illness... would you like me to give it to you straight?' He said Yes. Then he was told 'You only have a few months left to live'. Another one is without confirming of patients expect regarding life prognosis disclosure.

Unable to accept the doctor's blame for the delay in the initial hospital visit

The patient found it difficult to accept the doctor's words such as 'Why didn't you come a month earlier?'. Being told what he should have done did not help the patient.

Uncomfortable with the usage of inappropriate metaphors

The doctor used a strange metaphor to refer to the diagnosis, 'an unwelcome present for the New Year', which did not click with the patient and left him uncomfortable.

Pessimistic thoughts despite optimistic explanations

Even when affirmative words were used in the explanation, the patient still had pessimistic thoughts. For 
Box 1 Negative recollections of patients about doctor's words/attitudes regarding disclosure of cancer diagnosis $(n=17)$

[Surprised at the abrupt disclosure of the diagnosis]

$\checkmark$ It was first said to me when the doctor put his hand in for the palpation [...] and he said, "This, um, may be cancer". I was really shocked; Dx75(2005), Int77(2007)

$\checkmark$ I was told, "I'd say that a case like this is already terminal. [...] such a sudden delivery of that news really took me off guard; Dx57(2005), Int60(2008)

[Displeased by the direct disclosure of the diagnosis to the patient in the absence of family members]

$\checkmark$ They just said, "You've got cancer," matter-of-factly [...] Usually they call your wife in, or tell your children first in secret without the patient knowing. That was the type of world I was expecting, but instead they just said it directly to me, and I thought "W-What?"; Dx64(2001), Int71(2008)

[Unable to accept the doctor's negative words in the explanations] $\checkmark$ When I was told I have "bad-looking cancer," I began to feel hopeless; Dx73(2005), Int 76 (2008)

$\checkmark$ They said it was very bad, and well, undifferentiated, or not very differentiated. [...] I was really shocked at that time; Dx73(2005), Int76(2008)

$\checkmark$ The Gleason score is a measure of how it looks, and a score of $4+5$ is quite bad. It was then that I first realised that I had very bad cancer. After that, I was shocked by a lot of other things too; Dx 50 (2006), Int 52 (2008)

$\checkmark$ It started out with, 5 and 4. That means it has a Gleason score of 9 , but this is the worst possible kind [...] They explained a lot to me at the time, though. I didn't quite come into my mind what the doctor explained. I was in shock [laughs], and I didn't really understand; Dx49(2005), Int53(2009)

$\checkmark$ Progressive terminal cancer. You have about... 6 months to live. [...] Of course I was in shock; Dx74(2001), Int81(2008)

$\checkmark$ They said that they ran some tests, and it was prostate cancer. Not only that, but if there were 4 levels, it was a $4^{\prime}$, which is a very serious figure. It was like a thunderbolt out of the blue; I was so shocked; Dx 57 (2007), Int 58 (2008)

$\checkmark I$ was always told really negative things like, "your tumour markers are 100x higher," or "your cancer is advanced, and malignant," and things like that, which was threatening. Yeah, that's why I hated going to see the doctor; Dx57(2005), Int60(2009)

[Unable to understand the doctor's technical jargon]

$\checkmark$ I was only told, "Your cancer is 3 plus 3, so 6. It is a highly differentiated cancer," but I did not know what that meant at all, and they would not explain it for me clearly, so all I understood was that it was early stage; Dx76(2006), Int78(2008)

$\checkmark$ They said that, "It has completely, kind of, um, infiltrated up to your seminal vesicle," but I couldn't make sense of what that meant; Dx75(2005), Int77(2007)

[Distrust due to failure in the diagnosis based on previous examinations]

$\checkmark$ When you take a CT scan, everything should be more or less visible. I heard that they were aiming there, but the cancer was on the other side, so I thought that I would definitely not take any more radiation. Yeah. It would cause me more trouble if they made mistakes; Dx66(2003), Int72(2008)

[Aggrieved at doctor's unwillingness to allow questions]

$\checkmark$ Instead of talking about the results, I was told, "Okay, please come back in this month on this day," and that was it. Then, when I tried to ask

Continued
Box 1 Continued

questions, they said, "Oh, um, take care, take care, take care," which was more like get out of here, get out of here, get out of here. I thought that was unacceptable, but what could I do? So, I decided to leave then; Dx71(2007), Int72(2008)

[Dissatisfied with explanations involving facts only]

$\checkmark$ What should we do (for treatment)? Instead of asking me what we should do, [...] I would have wanted some heartfelt words, or something more like that; Dx60(2007), Int61(2008)

$\checkmark$ I was told "You only have a few months to left to live." When I said, "Huh? What do you mean?" the doctor explained that, "The prostate cancer cells have already metastasised throughout your entire body." At the time, I just thought, 'huh?' It was a huge shock; Dx64(2001), Int71(2008)

[Indignant over the unexpected disclosure of life expectancy]

$\checkmark$ I was asked, "About your illness... would you like me to give it to you straight?" I didn't think anything of it (life prognosis disclosure) at the time, so I said "Yes, of course I want to know." Yes. Then I was told "You only have a few months left to live." [...] At the time, I just thought, 'huh?' It was a huge shock; (Dx 64 (2001), Int71(2008)

$\checkmark$ When I was notified about my cancer, I mentioned life expectancy, so I was told 'well, at this rate, the chance that you will be alive in 5 years is probably about 70\%' [...] I thought, why did they have to tell me that?; Dx65(2004), Int69(2009)

[Unable to accept the doctors' blame for the delay in the initial hospital visit]

$\checkmark$ I was told "If you came here a month ago, it would have been fine." What? It makes no difference to what I could do now (whether or not you say that now). Yeah, I had no idea about (the fact that I needed an urgent diagnosis); Dx64(2001), Int71(2008)

[Uncomfortable with the usage of inappropriate metaphors]

$\checkmark$ They said, "It's December 26, and this is my last job before the New Year. So, this is a New Year's present that you won't really want to accept, but here are your results, "But it didn't really occur to me what was meant by that; Dx77(2006), Int79(2008)

[Pessimistic thoughts despite optimistic explanations]

$\checkmark$...So, they only showed me one, and I was told that the cancer looked good. [...] I thought, "this happened to me 10 years earlier than it does for other people."; Dx61(2007), Int62(2008)

$\checkmark$ They explained that the cancer was described as not such a bad kind. [...] Of course, in my head the first thing I thought was that cancer kills you, and I had heard that it is incredibly painful; Dx76(2006), Int78(2008)

[ : : category names; Italicized text: narrations ineach category

Dx: Age at diagnosis of prostate cancer

Int: Age at interview

(): Christian era

instance, a patient who was told to have a 'good-looking cancer', still lamented his young age at diagnosis, or another who was stuck with the thought that 'cancer means dying painfully' despite being told 'your cancer is not that bad'.

\section{DISCUSSION}

This study was a qualitative secondary analysis of narrative prostate cancer patient data drawn from an existing database. We identified 11 categories of negative recollections 
by patients of the words/attitudes of doctors when receiving a cancer diagnosis.

First, we considered how cancer diagnoses should be delivered based on the recommendations in existing guidelines (SPIKES/SHARE). Of the 11 categories generated in this study, seven categories were sufficiently covered by the existing guidelines. For the category 'Surprised at the abrupt disclosure of the diagnosis', it is possible to avoid leaving patients with negative impressions by using words that prepare the patient in advance for the news. Similarly, for 'Displeased by the direct disclosure of the diagnosis to the patient in the absence of family members', the patient should be asked in advance about their preference for how a cancer diagnosis should be delivered. For 'Unable to accept the doctor's negative words in the explanations', it is necessary to avoid words with a particularly negative connotation. For 'Unable to understand the doctor's technical jargon', such technical jargon should be avoided, and the doctor should begin with a vocabulary level suited to the patient's level of understanding. For 'Distrust due to failure in the diagnosis based on previous examinations', the doctor should periodically verify the patients' comprehension. For 'Aggrieved at doctor's unwillingness to allow questions', the doctor should create sufficient opportunities for patients to ask questions, and in the case of 'Dissatisfied with explanations involving facts only', listen to the patient's worries and cares. The remaining four categories are not listed in SPIKES or SHARE. They pertain to clinical implications that should be adopted in practice. The first category is 'Indignant over the unexpected disclosure of life expectancy'. There is no reference in SPIKES or SHARE about inquiring whether or not the patient would like to know their life expectancy. In this study, the patient had impressions of resentment, thinking, 'Why did he have to say something like that?' in response to receiving the explanation that he 'had about a $70 \%$ chance of being alive in 5 years'. Previous reports have indicated that, in Japan, the proportion of patients who would like to know their life expectancy is $50.4 \%,{ }^{25}$ whereas in USA it is approximately $80 \% .^{34}$ As such, doctors should be aware that Japanese patients may not want to know their life expectancy. When first delivering a cancer diagnosis, it is necessary to limit the disclosure of life expectancy to only those cases in which the patient has explicitly confirmed their desire to know. There is much discussion surrounding how patients should convey their wishes to be informed of their life expectancy. Girgis et al recommend avoiding a definite time scale. ${ }^{35}$ Sato et al pointed out that the consistent problem underlying the negative impact of prognostic disclosure was the absence of shared understanding between doctors and patients regarding its objective. ${ }^{15}$ Listening to patients' reasons for concerns about life expectancy and conveying it in a way that suits the individual patient is one approach. The second category is 'Unable to accept the doctor's blame for the delay in the initial hospital visit'. It is clearly not ideal for a doctor to use such language or have such attitudes. Doctors should consider the effect that their words will have on their patients before actually uttering them and should avoid negative statements about the past that cannot be undone. The third category is 'Uncomfortable with the usage of inappropriate metaphors'. Careful usage of metaphors should be practised. It is necessary to avoid inappropriate metaphors and choose words that are acceptable to the patient. The final category is 'Pessimistic thoughts despite optimistic explanations'. Even when the doctor explained that he had a 'goodlooking cancer', the patient was not consoled and kept on lamenting about the early age of his diagnosis. Another patient was so obsessed with the idea that 'cancer means dying painfully' that the words of encouragement such as 'cancer is not that bad' did not help. Not only the manner of disclosing the cancer diagnosis, but also the patient's understanding of the term 'cancer', could influence how the doctor's words/attitudes are received on disclosure of a cancer diagnosis. Even when doctors judge the case to be less malignant from a medical point of view, they should bear in mind that the patient may take things more gravely. It is important to understand their words, right after breaking the bad news.

Next, we consider the doctor-patient relationship in Japan, where this study was conducted. In a survey of Japanese outpatients regarding how well patients conveyed their questions and opinions to doctors, about half of the respondents answered that 'They conveyed them satisfactorily', and the other half said that they 'partially conveyed them'. ${ }^{36}$ A patient receiving a cancer diagnosis is likely to be more disoriented than a general outpatient, so the chances that they do not sufficiently convey their questions and opinions are expected to be higher. The 11 categories of the negative reception of cancer diagnoses extracted in this study may be partially attributed to patients not expressing themselves and being left with distrust and discontentment without having these emotions resolved. It is difficult for doctors to understand the emotions and experiences of patients who are uncomfortable speaking about these things. Doctors need to bear in mind that not asking questions is not equivalent to not having questions, and a lack of emotional response does not necessarily indicate the absence of strong emotions. Doctors should seek verification from patients whether their misgivings and questions were adequately addressed. It is important to create an opportunity for patients to raise questions, or to voice their feelings of discontentment. The issue of being unable to freely ask questions to doctors is not unique to Japan. A German report suggested that the ongoing opportunity to ask questions should be added to SPIKES. ${ }^{37}$ Since it is common across many different countries for patients to have difficulty asking questions to doctors, this may be one of the causes of the various negative recollections seen in this study. It is possible that patients in other countries are also left with negative recollections of the words/attitudes of 
their doctors at the time their cancer diagnosis was delivered. Future studies may focus on elucidating the differences between different countries and cultures.

Third, we considered the change in doctor-patient communication about cancer diagnosis over time. Cancer notifications in Japan around 1990 were as low as about $20 \%$. Since then, the cancer notification rate increased to approximately $60 \%$ in $2005,73.5 \%$ in $2012,{ }^{38}$ and $92 \%-94 \%$ in $2016 .{ }^{39}$ The diagnoses era of patients with negative memories was between 2001 and 2007. In other words, this was after SPIKES (developed in 2000) but before SHARE (developed in 2001). It is unclear how consistently SPIKES was being applied in clinical practice. Moreover, the style of communication between doctors and patients appears to be different from that in 2019.

As a finding of this study, 7 of the 11 categories were sufficiently covered by the existing guideline (SPINES or SHARE), while the remaining four categories are not listed in SPIKES or SHARE. In Japan of 2019, almost all oncologist knew SPIKES or SHARE. As such, future research focusing on the negative recollections of doctor's words/attitudes regarding disclosure of cancer diagnosis is necessary.

\section{Limitations of this study and future outlook}

This study was a qualitative secondary analysis of narrative data. It should be noted that the interviews were not focused on patients' acceptance of their doctors' explanations/behaviours when receiving their cancer diagnosis. Thus, one limitation was that the patients' negative recollections of the doctors' words/attitudes might not have reached theoretical saturation. If the research was initially focused specifically on the negative experiences, patients would have likely spoken even more about a diverse range of negative experiences. Second, we did not investigate whether the words/attitudes attributed to the doctors by the patients were in fact accurate representations. This implies that the words/attitudes might possibly be due to the inaccurate recollection by patients. This study, rather than obtaining the facts, was focused on how patients interpreted and remembered the receiving of their cancer diagnoses. Third, the time frame from diagnosis to interview ( $1-7$ years) might have been affected by recall of the memories surrounding the cancer diagnosis. Fourth, patients' preferences regarding 'the delivery of bad news' may be influenced by their cultural background. ${ }^{20-22}$ However, the narrative data in this study were only about Japanese prostate cancer patients. Therefore, the findings could not be generalised to patients with other forms of cancer from Western countries. Finally, in order to appropriately implement uniform guidelines such as SPIKES and SHARE for all cancers in clinical practice, non-traditional sources of data such as patient narratives should be utilised, which may improve patientdoctor communications not just for prostate cancer but for other cancer types as well.

\section{CONCLUSION}

It is clear that patients have recollections of a variety of negative experiences regarding the words/attitudes of their doctors at the time that they received their prostate cancer diagnosis. The use of narrative data would facilitate the appropriate application of commonly used guidelines for the delivery of cancer diagnoses tailored to individual patients in clinical practice.

Contributors MT conducted the main analysis and authored this paper. MU checked the validity of the data extraction range and the coding. MU and TN jointly investigated the validity of the analysis based on a review of MT's results. In the event that their opinions did not match during the analysis process, these three researchers held discussions until the matter was resolved. SK verified the progress of the study, supervised the analyses and conducted a final check of the paper.

Funding The authors have not declared a specific grant for this research from any funding agency in the public, commercial or not-for-profit sectors.

Competing interests Dr Nakayama is the vice chief director of DIPEx-Japan. DIPEX-Japan is a non-profit organisation (NP0), which does not receive any funding from corporations, so it does not have any corporate interests. For this study, a total of 100,000 yen was paid to DIPEx-Japan, an accredited NP0, from 2016 to 2017 for data sharing fees.Dr Nakayama reports personal fees from Ohtsuka Pharamaceutical co., Nakamura hospital, Japan Medical Data Centre, Dainippon Sumitomo Pharmaceutical co., Ono Pharamaceutical co., Chugai Pharamaceutical co., Dentsu co., Takeda Pharamaceutical co., from Novo Nordisk Pharma. co., Jansen Pharma. co., Boehringer Ingelheim International GmbH, HANSHIN Dispensing Holding Co., Ltd., Pfizer Japan Inc., and Nikkei Business Publications, Inc., outside the submitted work.

Patient consent for publication Not required.

Ethics approval Ethical approval for this study was obtained from the Kyoto University Graduate School and Faculty of Medicine, Ethics Committee (approval number: R0492), and from DIPEx-Japan Ethics Committee (approval number: 7)

Provenance and peer review Not commissioned; externally peer reviewed.

Data availability statement Data may be obtained from a third party and are not publicly available.

Open access This is an open access article distributed in accordance with the Creative Commons Attribution Non Commercial (CC BY-NC 4.0) license, which permits others to distribute, remix, adapt, build upon this work non-commercially, and license their derivative works on different terms, provided the original work is properly cited, appropriate credit is given, any changes made indicated, and the use is non-commercial. See: http://creativecommons.org/licenses/by-nc/4.0/.

ORCID iD

Masako Torishima http://orcid.org/0000-0001-8152-5412

\section{REFERENCES}

1 Ferlay J, Colombet M, Soerjomataram I, et al. Estimating the global cancer incidence and mortality in 2018: GLOBOCAN sources and methods. Int J Cancer 2019;144:1941-53.

2 WHO International agency for research on cancer. Cancer today: population fact sheets: world. Available: https://gco.iarc.fr/today/ data/factsheets/populations/900-world-fact-sheets.pdf [Accessed 13 Oct 2019].

3 Kimura T, Egawa S. Epidemiology of prostate cancer in Asian countries. Int J Urol 2018;25:524-31.

4 Liu Y, Uemura H, Ye D, et al. Prostate cancer in Asia: design of a patient registry to inform real-world treatments, outcomes, and quality of life. Prostate International 2019;7:108-13.

5 Ock M, Choi WJ, Jo M-W. Trend analysis of major cancer statistics according to sex and severity levels in Korea. PLoS One 2018;13:e0203110.

6 Katanoda K, Hori M, Matsuda T, et al. An updated report on the trends in cancer incidence and mortality in Japan, 1958-2013. Jpn J Clin Oncol 2015;45:390-401.

7 WHO International agency for research on cancer. Cancer today: population fact sheets: Japan. Available: https://gco.iarc.fr/today/ data/factsheets/populations/392-japan-fact-sheets.pdf [Accessed 13 Oct 2019]. 
8 Cancer statistics in japan. Cancer statistics in japan '18: 9 fiveyear Survival Rate, Data from Population-based Cancer Registries (Diagnosed in 2006-2008). Available: https://ganjoho.jp/en/ professional/statistics/brochure/2018_en.html [Accessed 13 Oct 2019].

9 Wall DP, Kristjanson LJ, Fisher C, et al. Responding to a diagnosis of localized prostate cancer: Men's experiences of normal distress during the first 3 postdiagnostic months. Cancer Nurs 2013;36:E44-50.

10 Chambers SK, Hyde MK, Laurie K, et al. Experiences of Australian men diagnosed with advanced prostate cancer: a qualitative study. BMJ Open 2018;8:e019917.

11 Cohen $\mathrm{H}$, Britten N. Who decides about prostate cancer treatment? A qualitative study. Fam Pract 2003;20:724-9.

12 O'Shaughnessy PK, Laws TA, Esterman AJ. The prostate cancer journey: results of an online survey of men and their partners. Cancer Nurs 2015;38:E1-12.

13 Collaço N, Rivas C, Matheson L, et al. Prostate cancer and the impact on couples: a qualitative metasynthesis. Support Care Cancer 2018;26:1703-13.

14 Chambers SK, Hyde MK, Ip DF-K, et al. Systematic review of research into the psychological aspects of prostate cancer in Asia: what do we know? Asian Pac J Cancer Prev 2013;14:2621-6.

15 Sato RS, Beppu H, lba N, et al. The meaning of life prognosis disclosure for Japanese cancer patients: a qualitative study of patients' narratives. Chronic IIIn 2012;8:225-36.

16 Stewart MA. Effective physician-patient communication and health outcomes: a review. CMAJ 1995;152:1423-33.

17 Takayama T, Yamazaki Y, Katsumata N. Relationship between outpatients' perceptions of physicians' communication styles and patients' anxiety levels in a Japanese oncology setting. Soc Sci Med 2001;53:1335-50.

18 Street RL. How clinician-patient communication contributes to health improvement: modeling pathways from talk to outcome. Patient Educ Couns 2013;92:286-91.

19 Baile WF, Buckman R, Lenzi R, et al. SPIKES-A six-step protocol for delivering bad news: application to the patient with cancer. Oncologist 2000;5:302-11.

20 Baile WF, Lenzi R, Parker PA, et al. Oncologists' Attitudes Toward and Practices in Giving Bad News: An Exploratory Study. JCO 2002;20:2189-96.

21 Searight HR, Gafford J. Cultural diversity at the end of life. issues and guidelines for family physicians. Am Fam Physician 2005;71:515-22.

22 Shahidi J. Not telling the truth: circumstances leading to concealment of diagnosis and prognosis from cancer patients. Eur $J$ Cancer Care 2010;19:589-93.
23 Japan Psycho-Oncology Society. Communication skill training text: share Ver3.3, 2018. Available: http://www.share-cst.jp/02.htm [Accessed 2 Jun 2019].

24 Fujimori M, Akechi T, Akizuki N, et al. Good communication with patients receiving bad news about cancer in Japan. Psychooncology 2005;14:1043-51.

25 Fujimori M, Akechi T, Morita T, et al. Preferences of cancer patients regarding the disclosure of bad news. Psychooncology 2007;16:573-81.

26 Fujimori M, Parker PA, Akechi T, et al. Japanese cancer patients' communication style preferences when receiving bad news. Psychooncology 2007;16:617-25.

27 Back AL, Arnold RM, Baile WF, et al. Approaching difficult communication tasks in oncology. CA Cancer J Clin 2005;55:164-77.

28 Heaton J. Reworking qualitative data. London Sage Publication 2004:19-34.

29 Shi L. Health services research methods. Delmar Publishers 1997:114-23.

30 Ziebland S, McPherson A. Making sense of qualitative data analysis: an introduction with illustrations from DIPEx. Med Educ 2006;40:405-14.

31 Pope C, Ziebland S, Mays N. Qualitative research in health care: analysing qualitative data. BMJ 2000;320:114-6.

32 Lincoln YS, Guba EG. Naturalistic inquiry. Sage Publications, 1985: 332-56.

33 Braun V, Clarke V, et al. Thematic Analysis. In: Cooper H, Camic PM, Long DL, et al, eds. Apa Handbook of research methods in psychology, vol 2: research designs: quantitative, qualitative, neuropsychological, and biological. Washington DC, US: American Psychological Association, 2012: 57-71.

34 Kaplowitz SA, Campo S, Chiu WT. Cancer patients' Desires for communication of prognosis information. Health Commun 2002;14:221-41.

35 Girgis A, Sanson-Fisher RW. Breaking bad news: consensus guidelines for medical practitioners. JCO 1995;13:2449-56.

36 Ministry of Health, Labour and Welfare. Patient's behavior survey, 2017. Available: https://www.mhlw.go.jp/toukei/saikin/hw/jyuryo/17/ index.html [Accessed 1 May 2019].

37 Seifart C, Hofmann M, Bär T, et al. Breaking bad news-what patients want and what they get: evaluating the spikes protocol in Germany. Ann Oncol 2014;25:707-11.

38 Ministry of Health, Labour and Welfare. Trend of cancer notification rate in Japan. The 42th cancer control Council in Japan. Council minutes in 14 February 2014.

39 National Cancer Centre: Cancer information centre and cancer registry centre. Reports of all Japan statistics, 2016. Available: https://ganjoho.jp/data/reg_stat/statistics/brochure/2016_report.pdf [Accessed 13 Oct 2019]. 